\title{
Genetic and clinical characteristics in Japanese hereditary breast and ovarian cancer: first report after establishment of $\mathrm{HBOC}$ registration system in Japan
}

\author{
Masami Arai ${ }^{1}$ Shiro Yokoyama ${ }^{2} \cdot$ Chie Watanabe ${ }^{3} \cdot$ Reiko Yoshida $^{1} \cdot$ Mizuho Kita $^{1} \cdot$ Megumi Okawa $^{4}$. \\ Akihiro Sakurai $^{5} \cdot$ Masayuki Sekine $^{6} \cdot$ Junko Yotsumoto $^{7} \cdot$ Hiroyuki Nomura $^{8} \cdot$ Yoshinori Akama $^{9}$. \\ Mayuko Inuzuka ${ }^{2} \cdot$ Tadashi Nomizu $^{10} \cdot$ Takayuki Enomoto $^{6} \cdot$ Seigo Nakamura $^{2}$
}

Received: 9 May 2017 / Revised: 17 August 2017 / Accepted: 18 August 2017 / Published online: 8 November 2017

(c) The Author(s) 2018. This article is published with open access

\begin{abstract}
The hereditary breast and ovarian cancer (HBOC) registration system of Japan was established by the Japanese HBOC Consortium. The first trial was registered in 2015 in four institutions to which some registration committee members belonged. We analyzed the information of 830 Japanese pedigrees, who underwent BRCA1/2 genetic testing, including mutation carriers with $B R C A 1(N=127)$ and $B R C A 2(N=115)$, and their families. The mutation-positive rate was $19.7 \%$. Variants of uncertain significance were found in $6.5 \%$ of all individuals subjected to genetic testing for BRCA1/2. Compared to the United States, Japan had a higher mutation-positive rate in most categories, except for the groups with male breast cancer. Among the intrinsic subtypes of $B R C A l$-associated breast cancers, $75.8 \%$ were triple-negative. The incidence rate of contralateral breast cancer in BRCA1/2 mutation carriers was 0.99\%/year. Among 240 mutation carriers, 26 and 62 patients underwent risk-reducing mastectomy (RRM) and risk-reducing salpingo-oophorectomy (RRSO), respectively; the respective frequencies of occult cancer were 7.1 and 3.2\%. Metachronous breast cancer after RRM or peritoneal cancer after RRSO was not observed during the follow-up period. The nationwide registration system began last year and the system enables followup analysis in Japan.
\end{abstract}

\section{Introduction}

Hereditary breast and ovarian cancer syndrome (HBOC) is an autosomal dominant inherited cancer susceptibility disorder caused by deleterious germline mutations in BRCAI

Electronic supplementary material The online version of this article (https://doi.org/10.1038/s10038-017-0355-1) contains supplementary material, which is available to authorized users.

Masami Arai

marai@jfcr.or.jp

1 Clinical Genetic Oncology, Cancer Institute Hospital of Japanese Foundation for Cancer Research, Tokyo, Japan

2 Breast Center, Showa University, Tokyo, Japan

3 Faculty of Human Science, Sophia University, Tokyo, Japan

4 St Luke's International Hospital, Tokyo, Japan

5 Department of Medical Genetics, Sapporo Medical University, Hokkaido, Japan or BRCA2 (BRCA1/2). Starting in 2004, BRCAl/2 genetic testing became a part of laboratory examinations in Japan. However, medical insurance does not currently cover the clinical practice of $\mathrm{HBOC}$, including genetic testing for BRCA1/2, medical surveillance, and preventive surgeries. Therefore, these procedures are not prevalent in routine clinical practice, and the clinical and pathological characteristics of HBOC in Japan remain unclear.

International database systems such as CIMBA (the Consortium of Investigators of Modifiers of BRCA1/2) or

6 Department of Obstetrics and Gynecology, Niigata University Graduate School of Medical and Dental Science, Niigata, Japan

7 Natural Science Division, Faculty of Core Research, Ochanomizu University, Tokyo, Japan

8 Department of Obstetrics and Gynecology, Keio University School of Medicine, Tokyo, Japan

9 Clinic of Familial Tumor, Hoshi General Hospital, Fukushima, Japan

10 Department of Surgery, Hoshi General Hospital, Fukushima, Japan 
Table 1 Registration items in the HBOC registration system

Basic information for the subject or other family members

1. Family relationship, 2.Gender, 3. Race

4. Father's individual number, 5. Mother's individual number (number issued by this system)

6. Date of birth ${ }^{\mathrm{a}}$

7. Date of death, 8. Age at death, 9. Cause of death, 10. History of cancers

11. Menopausal state, 12. Menopausal age, 13. Age at menarche, 14. Times of childbirth, 15. Age at first childbirth

Information on breast cancer ${ }^{\mathrm{b}}$

16. Onset age of breast cancer

17. Single or multiple, unilateral or bilateral, 18. Site (right or left), distant metastasis

19. Modality of diagnosis (trigger of finding a lesion)

20. Medical treatment (surgery, preoperative chemotherapy, post-operative chemotherapy, preoperative hormone therapy, postoperative hormone therapy, molecular-targeted drug, radiotherapy, no treatment)

21. Operation procedure, 22. Operation date

23. Histology, 24. Pathological tumor size (clinical tumor size), 25. Number of lymph node positive for cancer, 26. ER, 27. PGR, 28. HER2 (IHC), 29. HER2 (FISH)

30. Ki67 score, 31. Epithelial marker, 32. Nuclear grade (NG)

Information on ovarian cancer

33. Onset age of ovarian cancer

34. Ovarian cancer, tubal cancer, peritoneal cancer

35. Histology, 36. Stage, 37. Degree of differentiation for cancer, 38. Metastatic sites

39. Date medical treatment was started, 40. Operation date, 41. Radicality

42. Date of detection of first recurrence

Effect of chemotherapy for ovarian cancer

43. Preoperative chemotherapy, 44. Regimen, 45. Best effect

Post-operative chemotherapy ${ }^{\mathrm{c}}$

46. Regimen, 47. Period from completion of the last regimen to the recurrence, 48. Best effect, 49. Progression-free period

Other cancers than breast and ovary (all cancers should be described if patients have a history of plural cancers)

50. Type of other cancers, 51. Onset age

52. Other history associated with HBOC

Information on genetic tests

53. Date of genetic test for $B R C A$, 54.Type of examination, source of genetic test (company, medical institute, etc.), 55. Purpose of genetic test

56. Mutation of $B R C A 1$, Mutation site ${ }^{\mathrm{d}}$ (DNA and protein), 57. Mutation of $B R C A 2$, mutation site ${ }^{\mathrm{d}}$ (DNA and protein)

Information on prophylactic surgery of the breast and the ovary

58. Risk-reducing mastectomy (RRM), 59. Operation procedure for RRM, 60. Pathological findings of the resected specimen, 61. Execution age of RRM

62. Risk-reducing salpingo-oophorectomy (RRSO), 63. Operation procedure for RRSO, 64. Pathological findings of the resected specimen, 65. Execution age of RRSO

Information on visiting a hospital

66. First consultation at the hospital, 67. Final consultation at the hospital

Bold characteristics indicate essential registration items for all subjects. Italic characteristics indicate essential registration items for BRCA mutation carriers. No. 1, 16, 33, 50, and 51 are essential registration items for registrees within the second relatives of the subject or uncles, who suffered from any cancers and registered by interview-based information (they are defined as the Others in Table 2)

${ }^{a}$ The date is deleted at submission (only month and year are registered) for protection of individual information

${ }^{\mathrm{b}} \mathrm{We}$ can register up to the third breast cancer, if patients had suffered from it

${ }^{c}$ We can register up to the third regimen, if patients had received one

${ }^{\mathrm{d}}$ Based on the Myriad notation or HGVS notation 
Asian BRCA consortium about BRCA1/2 have already been established [1, 2]. However, data from Japanese HBOC families are poorly represented in these international databases or collaborative studies. Comparison of genetic and clinical features between other foreign countries and Japan cannot be performed in detail. The Korean Hereditary Breast Cancer study (KOHBRA) characterized clinical and genetic features [3]. Therefore, the Japanese nationwide registration system specific to $\mathrm{HBOC}$ is definitely required to clarify clinical and genetic features of Japanese HBOC and to improve medical treatment of HBOC.

The Japanese HBOC Consortium (JHC) was established in December 2012, with 342 members in December 2016. The aim of JHC is to spread awareness of HBOC and to provide effective healthcare for patients with HBOC and their families [4]. We established a registration committee for JHC in October 2013, and pushed it forward as a nationwide registration project. Before formal nationwide registration, we first performed trial registration in four institutions, which, in total, include over $40 \%$ of cases with BRCA1/2 genetic testing, to confirm that the electronic registration system and analytical system worked appropriately. Here, we report the results and issues of this registration system in $\mathrm{JHC}$.

\section{Materials and Methods}

\section{Subjects and registration}

The registered subjects were all Japanese individuals who underwent $B R C A 1 / 2$ genetic testing (including individuals in which no mutation was detected) from January 2012 to December 2014. We began the trial registration at the following four medical institutions, to which some of the registration committee members belonged: St. Luke's International Hospital (Tokyo), Cancer Institute Hospital (Tokyo), Showa University Hospital (Tokyo), and Hoshi General Hospital (Fukushima).

This study was approved by the Ethical Review Board of JHC in December 2014. After further approval by the Ethical Review Board or Institutional Review Board of each medical institution, we began this registration project. Informed consent was obtained from all subjects. If informed consent could not be received face to face for retrospective cases for whom medical treatment had been terminated or for patients who died, the candidate or the candidate's family members could opt out on the homepages of JHC and each participating institute. All subjects received genetic counseling and genetic testing of their own free will in clinical practice, including recommendation for further evaluation by the doctor in charge. All genetic testing with sequence and large rearrangement analysis was performed at Myriad Genetic Laboratories or FALCO Biosystems. The detected variants were interpreted according to the criteria of Myriad Genetic Laboratories. Sequencing and large rearrangement analysis were ordered in all but 10 cases. Among them, only sequencing was performed even if pathogenic mutations were not detected by sequencing.

For each subject, we also collected data on history of any cancers within second-degree relatives and in cousins. We entered information for BRCA1/2 genetic testing and the clinical and pathological findings of breast cancer, ovarian cancer, and other cancers in the original electronic template, which was referred to the previous Japanese research (Supplementary Fig. 1; Table 1) [5]. All data except sex were anonymously registered in each institution. The date of birth only included the year and month. The date was registered as 15 in all cases. Input batch data were sent to the datacenter together, which is located in Showa University Hospital. Data cleaning was applied to resolve defects of data, if necessary. We attempted to fill the template as completely as possible.

\section{Prevalence of $B R C A 1 / 2$ mutations}

On the basis of the affected risk of $B R C A 1 / 2$ mutation, we prepared a prevalence table and compared the rate of each condition with that previously reported by Sugano et al. [6] As with the data for the USA, we referred to the report by Frank et al. [7] The nomenclature of the gene variants was based on both the Myriad notation (nucleotide number is described according to GenBank accession number U14680 for BRCAl and U43746 for BRCA2) and the HGVS standard notation [8].

\section{Tumor spectrum and clinical and pathological features of BRCA-related breast cancer}

Based on the registered clinical data, we examined what types of cancer were frequently observed in Japanese $B R C A 1 / 2$ mutation carriers, and intrinsic subtypes of breast cancers in BRCAl/2 mutation carriers based on ER, PgR, and HER2 status. Age of onset of cancer is one of the most important characteristics. We analyzed the difference in the onset age of breast cancer among the three groups using one-way analysis of variance, and results were considered statistically significant at $P<0.05$. To analyze the difference in the onset age of ovarian cancer, we performed statistical analysis using the Student's $t$ test, and results were considered statistically significant at $P<0.05$. 
Table 2 Characteristics of Japanese registrees
Male Female Average age at BRCA1/2 genetic test (youngest-oldest)

\begin{tabular}{|c|c|c|c|c|}
\hline Pedigree & 830 & & & \\
\hline Registrees $^{\mathrm{a}}$ & 3867 & 1176 & 2691 & \\
\hline Subjects $^{\mathrm{b}}$ & 944 & 22 & 922 & $46.9(20-85)$ \\
\hline Others $^{c}$ & 2923 & 1154 & 1769 & \\
\hline $\begin{array}{l}\text { Clients who received BRCA } 1 / 2 \\
\text { genetic testing }\end{array}$ & 963 & 23 & 940 & $47.0(20-85)$ \\
\hline$B R C A 1$ mutation positive & 127 & 5 & 122 & $45.0(23-79)$ \\
\hline$B R C A 2$ mutation positive & 115 & 4 & 111 & $45.4(23-74)$ \\
\hline
\end{tabular}

${ }^{a}$ Registrees consisted of subjects and the others

${ }^{\mathrm{b}}$ Clients who provided informed consent at the time of registration in any of the four participating medical institutes

${ }^{c}$ Registrees within the second relatives of the subjects or uncles, who suffered from any cancers and registered by interview-based information

\section{Incidence of contralateral breast cancer}

Regarding the incidence rate of contralateral breast cancer, we calculated the frequency of the onset of contralateral breast cancer using the person-year method. We defined the observation period as the term between the day on which a registree received $B R C A 1 / 2$ genetic testing and the latest day that we confirmed her condition at each institute. We calculated the incidence rate of breast cancer or ovarian cancer in unaffected $B R C A 1 / 2$ mutation carriers in the same manner.

\section{Risk-reducing surgery}

The status of risk-reducing surgery, including risk-reducing mastectomy (RRM) and risk-reducing salpingo-oophrectomy (RRSO), is also presented. Clinical and pathological data were extracted from the registration database. We examined the frequency of secondary breast cancers and peritoneal cancers during the observation period and the frequency of occult cancers.

\section{Results}

\section{Subjects and registration}

Information on 846 families was collected from the four aforementioned medical institutions. We ascertained clinical and genetic characteristics of Japanese HBOC. Therefore, 13 foreign persons were excluded and 3 overlapping cases among two institutes were adjusted. Thus, we conducted the following analysis on 830 Japanese pedigrees. Among them, 944 individuals visited one of the four hospitals and provided informed consent for the registration project (22 males, 922 females, average age 46.9 years (20-85))
(Table 2). Therefore, we had 3867 registrees including 963 individuals who received $B R C A 1 / 2$ genetic testing (including those who underwent the testing outside of the four medical institutes). The deleterious BRCA1/2 mutation was observed in $161(19.7 \%)$ of 817 cases with no previous information on BRCAl and BRCA2 mutation status (BRCA1: 84, BRCA2: 76, both: 1; 13 cases already had $B R C A 1 / 2$ genetic test results in their pedigree). Thus, these cases were excluded in the analysis of prevalence rate. In total, $240 B R C A 1$ or $B R C A 2$ mutation carriers were registered in this study (BRCA1: 125, BRCA2: 113, both: 2). (Table 2).

\section{Prevalence of $B R C A 1 / 2$ mutations}

Pathological mutations of $B R C A 1$ and $B R C A 2$ were widely distributed across both whole genes (Table 3 ). Most deleterious mutations had been reported twice or less at the four institutions, but the L63X mutation in exon 5 of BRCAl was frequently encountered, and it thus might be a mutation hotspot in Japan. Fifty-three variants of uncertain significance (VUS) were detected in $6.5 \%$ of all genetic tests for BRCA1/2 (BRCA1: 21, BRCA2: 31, both: 1) (Table 4). In addition, there were eight VUS having simultaneous deleterious mutations in $B R C A 1 / 2$.

A prevalence table was generated based on the data from this trial registration. In most groups, except the male breast cancer group, the $B R C A 1 / 2$ mutation frequency was higher in Japanese individuals than that in Americans (nonAshkenazi Jews) (Table 5).

\section{Tumor spectrum and clinical and pathological features of BRCA-related breast cancer}

We examined what type of cancer occurred in BRCA1/2 mutation carriers. As expected, breast cancer was the most 


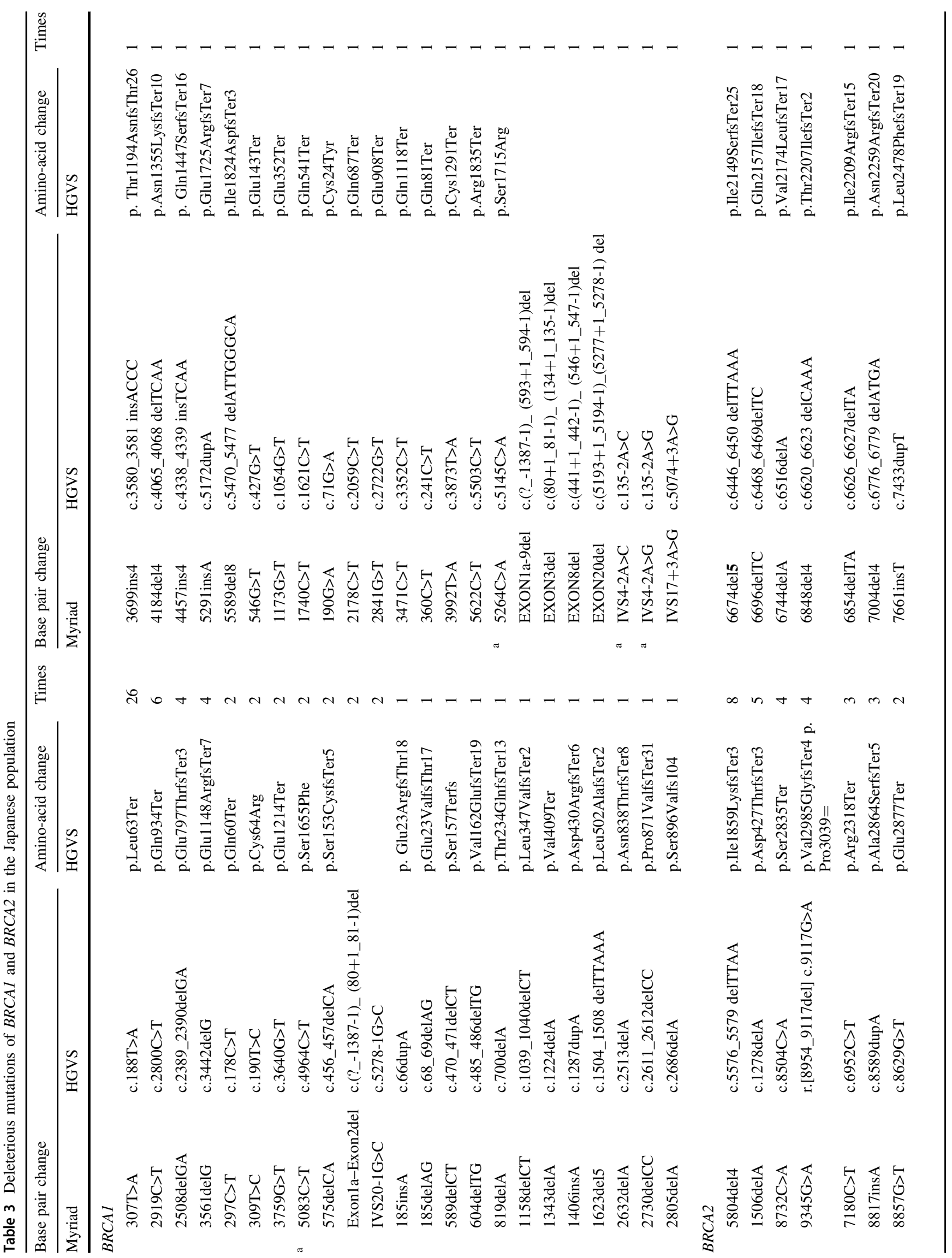




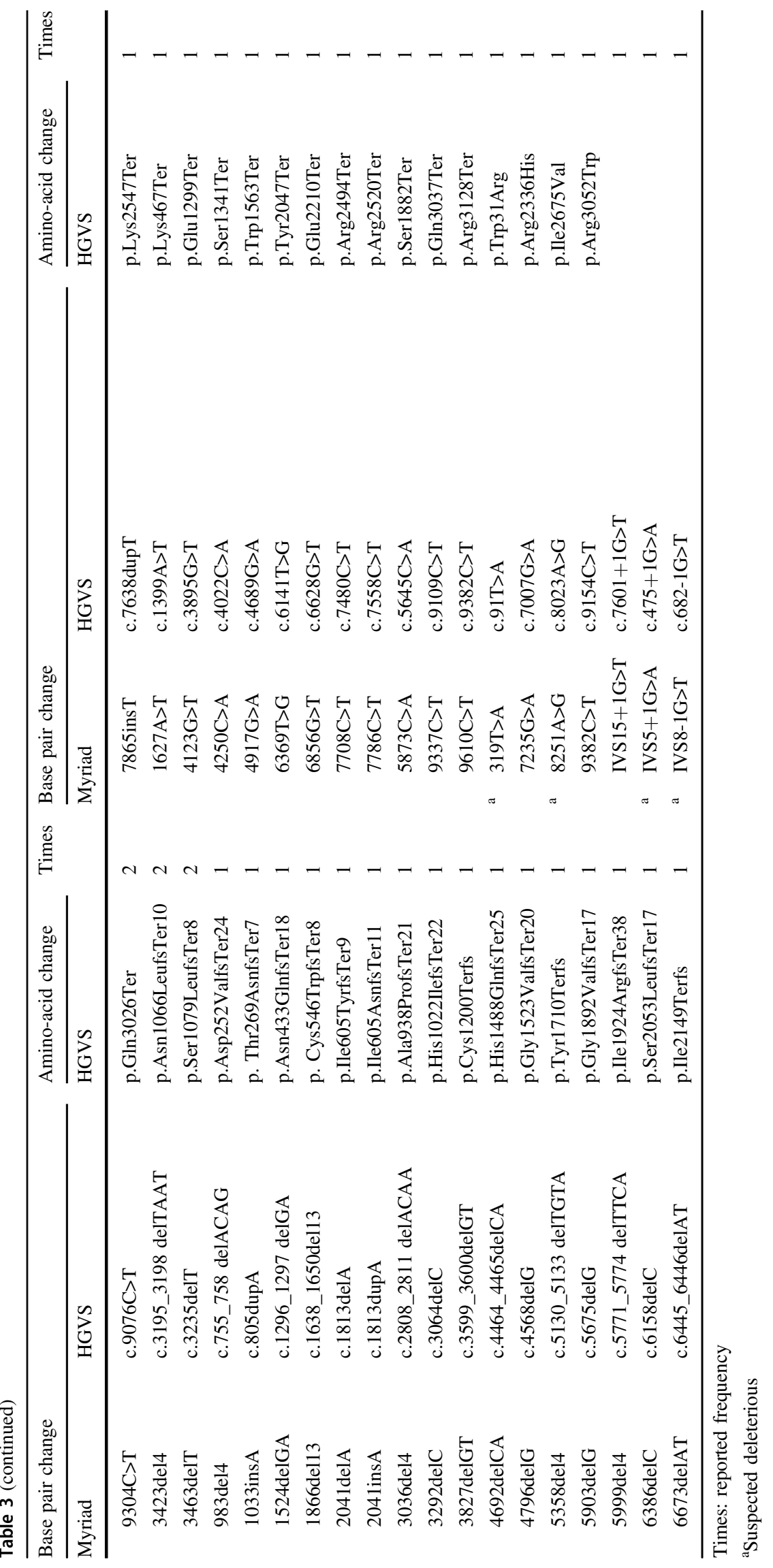


Table 4 Variants of uncertain significance (VUS) of BRCA1 and BRCA2 in the Japanese population

\begin{tabular}{|c|c|c|c|c|c|c|c|c|c|}
\hline \multicolumn{3}{|c|}{ Base pair change } & \multirow{2}{*}{$\frac{\text { Amino-acid change }}{\text { HGVS }}$} & \multirow[t]{2}{*}{ Times } & \multicolumn{3}{|c|}{ Base pair change } & \multirow{2}{*}{$\frac{\text { Amino-acid change }}{\text { HGVS }}$} & \multirow[t]{2}{*}{ Times } \\
\hline & 4yriad & HGVS & & & & yriad & HGVS & & \\
\hline \multicolumn{10}{|c|}{$B R C A 1$} \\
\hline & $273 \mathrm{C}>\mathrm{T}$ & c. $154 \mathrm{C}>\mathrm{T}$ & p.Leu52Phe & 3 & & $2683 \mathrm{~A}>\mathrm{C}$ & c. $2564 \mathrm{~A}>\mathrm{C}$ & p.Gln855Pro & 1 \\
\hline & $745 \mathrm{C}>\mathrm{T}$ & c. $626 \mathrm{C}>\mathrm{T}$ & p.Pro209Leu & 3 & a & $2845 \mathrm{~A}>\mathrm{T}$ & c. $2726 \mathrm{~A}>\mathrm{T}$ & p.Asn909Ile & 1 \\
\hline & $1321 \mathrm{G}>\mathrm{A}$ & c. $1202 \mathrm{G}>\mathrm{A}$ & p.Gly401Glu & 2 & & $3568 \mathrm{C}>\mathrm{G}$ & c. $3449 \mathrm{C}>\mathrm{G}$ & p.Pro1150Arg & 1 \\
\hline & $5677 \mathrm{~A}>\mathrm{G}$ & c. $5558 \mathrm{~A}>\mathrm{G}$ & p.Tyr1853Cys & 2 & & $4129 A>G$ & c. $4010 \mathrm{~A}>\mathrm{G}$ & p.Asp1337Gly & 1 \\
\hline \multirow[t]{4}{*}{$\mathrm{a}$} & $517 \mathrm{G}>\mathrm{A}$ & c. $398 \mathrm{G}>\mathrm{A}$ & p.Arg133His & 1 & & $4253 \mathrm{C}>\mathrm{T}$ & c. $4134 \mathrm{C}>\mathrm{T}$ & p.Val1378Val & 1 \\
\hline & Unknown & Unknown & p.Arg166Ser & 1 & & $4879 \mathrm{C}>\mathrm{T}$ & c. $4760 \mathrm{C}>\mathrm{T}$ & p.Ser1587Leu & 1 \\
\hline & $895 \mathrm{~A}>\mathrm{T}$ & c. $776 \mathrm{~A}>\mathrm{T}$ & p.Glu259Val & 1 & & $5054 \mathrm{G}>\mathrm{C}$ & c. $4935 \mathrm{G}>\mathrm{C}$ & p.Arg1645Ser & 1 \\
\hline & $1476 \mathrm{G}>\mathrm{C}$ & c. $1357 \mathrm{G}>\mathrm{C}$ & p.Glu453Gln & 1 & a & $5466 \mathrm{~A}>\mathrm{C}$ & c. $5347 \mathrm{~A}>\mathrm{C}$ & p.Met1783Leu & 1 \\
\hline \multirow[t]{2}{*}{ a } & $1998 \mathrm{G}>\mathrm{A}$ & c. $1879 \mathrm{G}>\mathrm{A}$ & p.Val627Ile & 1 & & IVS17-9A>G & c. $5075-9 A>G$ & & 1 \\
\hline & $2646 A>G$ & c. $2527 \mathrm{~A}>\mathrm{G}$ & p.Thr843Ala & 1 & & & & & \\
\hline \multicolumn{10}{|c|}{$B R C A 2$} \\
\hline $\mathrm{a}$ & $281 \mathrm{G}>\mathrm{A}$ & c. $53 \mathrm{G}>\mathrm{A}$ & p.Arg18His & 4 & & $6257 \mathrm{~T}>\mathrm{G}$ & c. $6029 \mathrm{~T}>\mathrm{G}$ & p.Val2010Gly & 1 \\
\hline a & $6197 \mathrm{~A}>\mathrm{C}$ & c. $5969 \mathrm{~A}>\mathrm{C}$ & p.Asp1990Ala & 4 & & $7777 \mathrm{~A}>\mathrm{G}$ & c. $7549 \mathrm{~A}>\mathrm{G}$ & p.Thr2517Ala & 1 \\
\hline a & $779 \mathrm{~T}>\mathrm{C}$ & c. $551 \mathrm{~T}>\mathrm{C}$ & p.Leu184Pro & 3 & & $7910 \mathrm{~A}>\mathrm{T}$ & c. $7682 \mathrm{~A}>\mathrm{T}$ & p.Gln2561Leu & 1 \\
\hline \multirow[t]{10}{*}{ a } & IVS3-10A $>G$ & c. $317-10 \mathrm{~A}>\mathrm{G}$ & & 3 & & $8633 \mathrm{C}>\mathrm{T}$ & c. $8405 \mathrm{C}>\mathrm{T}$ & p.Pro2802Leu & 1 \\
\hline & $851 \mathrm{~T}>\mathrm{G}$ & c. $623 \mathrm{~T}>\mathrm{G}$ & p.Val208Gly & 2 & & $8719 A>T$ & c. $8491 \mathrm{~A}>\mathrm{T}$ & p.Met2831Leu & 1 \\
\hline & $5082 \mathrm{~T}>\mathrm{A}$ & c. $4854 \mathrm{~T}>\mathrm{A}$ & p.Asp1618Glu & 2 & & $8927 \mathrm{~A}>\mathrm{G}$ & c. $8699 \mathrm{~A}>\mathrm{G}$ & p.Asp2900Gly & 1 \\
\hline & $324 \mathrm{~T}>\mathrm{G}$ & c. $96 \mathrm{~T}>\mathrm{G}$ & p.Phe32Leu & 1 & & $9394 \mathrm{C}>\mathrm{T}$ & c. $9166 \mathrm{C}>\mathrm{T}$ & p.His3056Tyr & 1 \\
\hline & $478 \mathrm{C}>\mathrm{G}$ & c. $250 \mathrm{C}>\mathrm{G}$ & p.Gln84Glu & 1 & & $10,049 \mathrm{~T}>\mathrm{G}$ & c. $9821 \mathrm{~T}>\mathrm{G}$ & p.Leu3274Trp & 1 \\
\hline & $2978 \mathrm{~T}>\mathrm{C}$ & c. $2750 \mathrm{~T}>\mathrm{C}$ & p.Val917Ala & 1 & a & 661del3 & c.433_435delGTT & p.Val145del & 1 \\
\hline & $3648 \mathrm{~T}>\mathrm{A}$ & c. $3420 \mathrm{~T}>\mathrm{A}$ & p.Ser1140Arg & 1 & & IVS5+9A $>T$ & c. $475+9 \mathrm{~A}>\mathrm{T}$ & & 1 \\
\hline & $4655 \mathrm{~A}>\mathrm{G}$ & c. $4427 \mathrm{~A}>\mathrm{G}$ & p.Asp1476Gly & 1 & & IVS6-12C $>\mathrm{A}$ & c. $517-12 \mathrm{C}>\mathrm{A}$ & & 1 \\
\hline & $5410 \mathrm{G}>\mathrm{A}$ & c. $5182 \mathrm{G}>\mathrm{A}$ & p.Asp1728Asn & 1 & & IVS6- $2 A>G$ & c. $517-2 \mathrm{~A}>\mathrm{G}$ & & 1 \\
\hline & $5545 \mathrm{G}>\mathrm{C}$ & c. $5317 \mathrm{G}>\mathrm{C}$ & p.Glu1773Gln & 1 & & IVS11-202 del20 & c.6842-202_6842-183del & & 1 \\
\hline
\end{tabular}

Times: reported frequency

${ }^{\mathrm{a}}$ Favor polymorphism

frequent followed by ovarian cancer and uterine cancer. Of 127 BRCA1 mutation carriers, 94 had a history of or were currently suffering from breast cancer (67 single cases, 24 cases with 2 simultaneously metachronous breast cancers, 3 with 3 simultaneous or metachronous cancers). Of 115 BRCA2 mutation carriers, 98 subjects had breast cancer (78 single cases, 18 cases with 2 breast cancers, 2 with 3 breast cancers). Ovarian cancers were observed in 14 cases (BRCA1; 9, BRCA2; 5). However, other cancers except endometrial cancer and cervical cancer were only reported once.

We examined intrinsic subtypes of breast cancer in BRCA1/2 mutation carriers based on the information about their ER, PgR, and HER2 status. Of 66 breast cancers in BRCA1 mutation carriers, $75.8 \%$ were triple-negative breast cancer (TNBC) and $21.2 \%$ were of the luminal type. Whereas of 59 breast cancers in BRCA2 mutation carriers, $18.6 \%$ were TNBC and $64.4 \%$ were luminal type, respectively.
The distribution of onset age for breast cancer and ovarian cancer was compared among BRCA1/2-positive and $B R C A 1 / 2$-negative groups. For first breast cancers, the average age of onset in the BRCA1- and BRCA2-positive groups (90 and 98 cases) and BRCA-negative group (588 cases) was 40.2 years (95\% confidence interval (CI) 38.15-42.25), 41.7 years (95\% CI 39.71-43.70), and 45.4 years (95\% CI 44.54-46.26), respectively. The onset age of breast cancer in the BRCAl-positive group was significantly lower than that of the $B R C A$-negative group $(P<0.001)$. A significant difference was also observed between the BRCA2-positive group and BRCA-negative group $(P=0.004)$. The difference regarding onset age of breast cancer between the BRCA1- and BRCA2-group was not statistically significant. For ovarian cancer, the average age of onset was 51.6 years (SD: 9.90) in the BRCAl/2 mutation-positive group and 44.5 years (SD: 10.38) in the mutation-negative group, but the difference in the onset age of ovarian cancer was not significant $(P=0.072)$; however, 
Table 5 Prevalence of deleterious mutations in BRCA1 and BRCA2 based on this study

\begin{tabular}{|c|c|c|c|c|c|c|}
\hline \multirow[t]{2}{*}{ Family history of breast cancer or ovarian cancer } & \multicolumn{4}{|c|}{ Among first- and second-degree relatives } & \multirow[t]{3}{*}{$\begin{array}{l}\text { No family history of breast } \\
\text { or ovarian cancer }\end{array}$} & \multirow[t]{3}{*}{$\begin{array}{l}\text { Family history in only } \\
\text { third-degree relatives }\end{array}$} \\
\hline & - & + & - & + & & \\
\hline Ovarian cancer (at any age) & - & - & + & + & & \\
\hline \multicolumn{7}{|l|}{ Patient's history } \\
\hline \multirow[t]{2}{*}{ Breast cancer $\geq 50 \mathrm{y}$} & $2 / 58$ & $9 / 58$ & $6 / 24$ & $1 / 8$ & $0 / 26$ & $0 / 8$ \\
\hline & $3.4 \%$ & $15.5 \%$ & $25.0 \%$ & $12.5 \%$ & $0 \%$ & $0 \%$ \\
\hline \multirow[t]{2}{*}{ Breast cancer $<50 \mathrm{y}$} & $21 / 125$ & $45 / 139$ & $27 / 61$ & $17 / 27$ & $16 / 164$ & $1 / 17$ \\
\hline & $16.8 \%$ & $32.4 \%$ & $44.3 \%$ & $63.0 \%$ & $9.8 \%$ & $5.9 \%$ \\
\hline \multirow[t]{2}{*}{ Ovarian cancer with no breast cancer at any age } & $0 / 2$ & $1 / 1$ & $1 / 2$ & - & $1 / 5$ & $0 / 1$ \\
\hline & $0 \%$ & $100 \%$ & $50.0 \%$ & - & $20.0 \%$ & $0 \%$ \\
\hline \multirow[t]{2}{*}{ Breast cancer and ovarian cancer at any age } & $1 / 1$ & $1 / 2$ & $4 / 4$ & - & $1 / 6$ & - \\
\hline & $100 \%$ & $50 \%$ & $100 \%$ & - & $16.7 \%$ & - \\
\hline \multirow[t]{2}{*}{ Male breast cancer at any age } & $0 / 1$ & $0 / 1$ & - & - & $0 / 1$ & - \\
\hline & $0 \%$ & $0 \%$ & - & - & $0 \%$ & - \\
\hline \multirow[t]{2}{*}{ No breast cancer or ovarian cancer at any age } & $0 / 16$ & $2 / 27$ & $3 / 20$ & $0 / 7$ & $1 / 4$ & $0 / 1$ \\
\hline & $0 \%$ & $7.4 \%$ & $15.0 \%$ & $0 \%$ & $25.0 \%$ & $0 \%$ \\
\hline
\end{tabular}

the onset age of ovarian cancer in the BRCA1/2-positive group tended to be higher than that in the $B R C A$-negative group. The number of cases with ovarian cancer was too small for a valid statistical analysis (14 and 15 cases in BRCA1/2 mutation-positive and -negative group, respectively).

The reason to receive genetic testing for BRCA1/2 was also registered. Personal healthcare was the most frequent answer, followed by facilitation of decision-making for an operation. Indeed, out of 50 mutation-positive cases, only 6 cases $(12.0 \%)$ chose breast conserving surgery, whereas of 218 mutation-negative cases, 120 cases (55.0\%) received breast conserving surgery.

\section{Incidence of contralateral breast cancer}

We also calculated the incidence rate of contralateral breast cancer development in patients with unilateral breast cancer. The average observation periods after the genetic tests were 27.3 and 16.2 months for BRCA1/2 mutation-positive and mutation-negative groups, respectively. Three contralateral breast cancers occurred in BRCA1/2 mutation carriers (3/ 302.6 person-years), and the annual incidence rate was $0.99 \%$. Breast cancer occurrence was observed in two BRCA1/2 non-mutation carriers (2/650.7 person-years, $0.33 \% / y e a r)$. The incidence of cancer in 37 unaffected BRCA1/2 mutation carriers was also examined. The mean observation period after genetic testing was 17.2 months (1-69 months). The average age at the time of genetic testing was 40.1 years (23-64 years) and, during the observation period, two incidences of breast cancer occurred (2/53.0 person-years). The incidence of breast cancer in unaffected $B R C A 1 / 2$ mutation carriers was $3.8 \% / y e a r$. The onset of ovarian cancer was not detected during this observation period in the same unaffected carriers.

\section{Risk-reducing surgery}

RRM and RRSO were performed in 26 and 62 patients, respectively, among 240 mutation carriers. All 26 RRM cases were classified as contralateral RRM. The average age at the RRM was 43.8 years (33-56 years). The average period from breast cancer onset to RRM was 2.46 years on average (0-14 years). Occult cancer was detected in two patients by post-operative pathological examination of RRM (7.7\%). Metachronous breast cancer from residual breast tissue was not detected during the observation period after RRM (mean observation period: 18.6 months (2-43.2 months)).

The average age at RRSO was 49.2 years (range, 36-65 years), and the mean interval from the onset of breast cancer was 5.1 years (except in five individuals without breast cancer). Two occult cancers (one fallopian tube cancer and one ovarian cancer) were detected upon pathological examination of the resected specimen (3.2\%). Peritoneal cancers were not observed during the follow-up period after RRSO (average observation period: 21.7 months (0-50.8 months)).

\section{Discussion}

Until recently, the actual state of clinical practice for HBOC in our country remained unclear. Information is not 
available on how risk-reducing surgeries are performed under the present medical insurance system in Japan or on how many cases of risk-reducing surgeries are performed. Our aim with this registration system is to clarify pathological and clinical features of HBOC among Japanese and to link authorized systems of medical institutes to improve awareness of HBOC in the future.

One of the merits of this registration system is that registrees, who received genetic testing for $B R C A 1 / 2$, including their family, can be followed up by annual update. The annual update of registration data enables us to perform prospective analysis and to improve data quality, providing revised clinical data. We collected HBOC data from four institutions that most actively work on HBOC clinical practice and perform extensive $B R C A 1 / 2$ genetic tests in Japan. No data on BRCA mutation carriers compared to the mutation-negative population are available in Japan at such a large scale. Approximately $40 \%$ or more of annual genetic $B R C A 1 / 2$ tests are ordered by these four institutions in Japan. Therefore, this trial registration would aptly reflect the characteristics of $B R C A 1 / 2$ mutations in the Japanese HBOC.

Nakamura et al. [5] showed deleterious mutations in $30.7 \%$ of patients with breast cancer and confirmed their clinical criterion for high risk. In the present registration, pathological mutations were confirmed in $19.7 \%$ of the subjects who first underwent genetic testing for $B R C A 1 / 2$ in their pedigrees. This lower prevalence rate might be explained as follows: first, in Nakamura's study, almost all subjects had personal history and family history of breast cancer and were more likely to be HBOC. In this registration, all subjects who received BRCA genetic testing were enrolled regardless of their personal history and family history. Second, even in Japan, as of late, genetic testing has become popular and widespread. One of the reasons is the so called "Angelina effect" [9]. Even patients without high risk of developing breast cancer might undergo genetic testing. Third, subjects who were enrolled in clinical examinations on PARP inhibitors were also included $(8.3 \%$ of all subjects). They were not always likely to be BRCA1/2 mutation carriers based on clinical characteristics and family history.

Mutations were distributed across almost all regions of both genes. Mutation frequency was particularly high for L63X in BRCA1, and this nonsense mutation seemed to be a hotspot in the Japanese population. Based on earlier studies, this mutation is a founder mutation by haplotype analysis, and carriers with the mutation are mainly from the eastern part of Japan [10, 11].

In the prevalence table, Japanese individuals had a higher mutation-positive rate for BRCA1/2 than Americans (except for Ashkenazi Jews) in almost all groups except for the male breast carcinoma groups. Sugano et al. [6] showed that the relative risk of $B R C A 1 / 2$ mutation in Japanese individuals is significantly higher than that in Americans (barring Ashkenazi Jews) (odds ratio $1.87, P=0.005$ ). The current study included more subjects and provided more reasonable and realistic results than the previous study. The mutation prevalence of $B R C A 1 / 2$ mutation carriers in Japanese individuals is higher than that in the USA except for the groups with male breast cancer [7]. Furthermore, our results seem to be more reasonable than the previous data. The prevalence rate of probands with breast cancer over 50 years of age, with no family history of early onset breast cancer and ovarian cancer, was $3.4 \%$ in our study and $2.3 \%$ in nonAshkenazi Americans, whereas it was $21.4 \%$ in the previous study $[6,7]$. Compared with the prevalence rate in the German consortium of $\mathrm{HBOC}$, the overall prevalence rate was similar to that in Germany. In the report, high mutation frequency was observed in families with two or more ovarian cancers $(41.9 \%)$, and families with one or more breast cancer and one ovarian cancer (41.6\%) [12]. According to our data, the prevalence rate of Japanese probands with early onset breast cancer and family history of ovarian cancer is also over $40 \%$. Family history of ovarian cancer is an important factor among studies in each country.

The frequency of TNBC is considered to be $10-15 \%$ among breast cancer subtypes [13]. The ratio of TNBC in individuals positive for BRCA1 mutations was $75.8 \%$ in this study. According to many reports, the frequency of TNBC in BRCAl mutation carriers is $\sim 60-80 \%$ [14]. CIMBA reported the frequency to be $68 \%$ [15].

The reason for receiving $B R C A 1 / 2$ genetic testing was also registered. The most common answer is that subjects received genetic testing for their own healthcare. Many of the mutation carriers received medical intervention such as regular medical checkups or risk-reducing surgeries under cooperation among the departments of breast surgery, gynecology, and clinical genetics.

The onset age of breast cancer in the BRCA1 or BRCA2 mutation-positive group was significantly lower than that in the $B R C A$ mutation-negative group (40.2 years, 41.7 years, and 45.4 years, respectively). This is also 20 years younger than that in the Japanese general population (60.8 years) $[16,17]$. Meanwhile, the average age of onset for ovarian cancer in the mutation-positive group, which is critical information for the appropriate age for RRSO, was approximately 7 years older than that of the mutationnegative group. However, this was still lower than that of the Japanese general population (61.1 years) [16, 17]. The tendency of older age of onset for ovarian cancer in BRCAI/ 2 mutation carriers could not be confirmed statistically because the number of cases with ovarian cancer was too low. National Comprehensive Cancer Network guidelines propose that $B R C A 1 / 2$ mutation carriers undergo RRSO at 
the age of 35-40 years [18]. The reasonable age for RRSO in Japan might be older than the age recommended by NCCN. RRSO is also recommended in the Japanese Breast Cancer Practice Guidelines. The appropriate age for RRSO should be considered in Japan as well [19].

The incidence rate of contralateral breast cancer in a BRCA1/2 mutation carrier was $0.99 \% / y e a r$, which was three times higher than that for a person without the mutation. However, the risk seems lower than that reported in previous foreign studies. Brekelmans et al. [20] reported that the risk of metachronous contralateral breast cancer was $3.1 \%$ year in both BRCA1 and BRCA2 mutation carriers. A meta-analysis of cumulative risks for contralateral breast cancer in BRCA1/2 mutation carriers with a first breast cancer showed a cumulative 10-year incidence rate of $27 \%$ and $19 \%$ for contralateral breast cancer in BRCAl and BRCA2 mutation carriers, respectively [21]. Therefore, further long-term and large-scale studies are required. In unaffected BRCAl/2 mutation carriers, two breast cancers, which occurred in patients in their 40s, were detected during the observation period of 17.2 months. The incidence rate of breast cancer is $3.8 \%$ year in unaffected BRCAl/2 mutation carriers.

Preventive surgeries are not yet widespread in Japan; RRM and RRSO were performed in 26 and 62 BRCA1/2 mutation carriers, respectively, at the four institutions studied. According to a recent report from overseas, preventive surgeries are being performed very frequently; $46 \%$ of BRCA1/2 mutation carriers underwent RRM and $70-80 \%$ of BRCA1/2 mutation carriers underwent RRSO [22]. However, a report indicated that RRSO is performed in $52.4 \%$ of cases in Korea, showing a slightly lower frequency compared to that in Europe or the USA [23]. In comparison to these countries, only a few people undergo preventive surgeries in Japan. The frequency of occult cancer in the resected specimens was 7.7\% in RRM and 3.2\% in RRSO. Evans et al. reported that occult cancers occurred in $2.3 \%$ of RRM cases, whereas reports from the USA and Korea showed that the incidence of occult cancers in RRSO is $5.4 \%$ and $11.7 \%$, respectively. Our data indicate a relatively low incidence despite the detailed pathological examination of resected ovaries and fallopian tubes. However, the sample size was small. Therefore, further studies are required to confirm these numbers [24, 25].

This study of the trial registration system to estimate clinical features of patients with HBOC presents some limitations. First, the medical staff who inputted data were largely specialized in breast cancer. Therefore, information about ovarian cancer was frequently lacking, particularly information about the regimen for chemotherapy and its effects, despite data cleaning. Additionally, we did not have sufficient data on ovarian cancers in BRCA mutation carriers. Furthermore, these data were collected from only four institutes and unevenly distributed within Tokyo or the greater Tokyo area. Therefore, the results do not reflect characteristics of the entire population of Japanese BRCA mutation carriers, although over $40 \%$ of clients receiving $B R C A 1 / 2$ genetic testing were covered in these four institutes.

Despite these limitations, we recognized the outline of clinical features of BRCA1/2 mutation carriers and clinical characteristics of breast and ovarian cancers with HBOC, and this trial registration elucidated some issues that should be addressed in the registration system described above.

In conclusion, we established a nationwide registration system for subjects receiving genetic testing for BRCA1/2 and started the official registration in March 2016. The registration system provides annual updated data reflecting the actual conditions for not only the subjects, but also relatives with cancer, an advantage of the Japanese registration system. In the future, it will be necessary to collect additional HBOC cases and to follow them up for a long period to clarify the genetic and clinical characteristics of HBOC in Japan.

Acknowledgements This work was supported by Health, Labour and Welfare Sciences Research Grants (H26-policy for cancer general012). We thank Dr. Kota Katanoda (Center for Cancer Control and Information Services, National Cancer Center), who kindly calculated and provided the average age of onset of female breast and ovarian cancers in the Japanese general population based on population-based cancer registry data.

\section{Compliance with Ethical Standards}

Conflict of interest The authors declare that they have no competing interests.

Open Access This article is licensed under a Creative Commons Attribution 4.0 International License, which permits use, sharing, adaptation, distribution and reproduction in any medium or format, as long as you give appropriate credit to the original author(s) and the source, provide a link to the Creative Commons license, and indicate if changes were made. The images or other third party material in this article are included in the article's Creative Commons license, unless indicated otherwise in a credit line to the material. If material is not included in the article's Creative Commons license and your intended use is not permitted by statutory regulation or exceeds the permitted use, you will need to obtain permission directly from the copyright holder. To view a copy of this license, visit http://creativecommons. org/licenses/by/4.0/.

\section{References}

1. Chenevix-Trench G, Milne RL, Antoniou AC, Couch FJ, Easton DF, Gokdgar DE. CIMBA. An international initiative to identify genetic modifiers of cancer risk in BRCA1 and BRCA2 mutation carriers: the Consortium of Investigators of Modifiers of BRCA1 and BRCA2 (CIMBA). Breast Cancer Res. 2007;9:104.

2. Nakamura S, Kwong A, Kim SW, Iau P, Patmasiriwat P, Dofitas $\mathrm{R}$, et al. Current status of the management of hereditary breast and ovarian cancer in Asia: first report by the Asian BRCA Consortium Public Health Genomics. 2016;19:53-60. 
3. Han SA, Park SK, Ahn SH, Lee MH, Noh DY, Kim LS, et al. The Korean Hereditary Breast Cancer (KOHBRA) study: protocols and interim report. Clin Oncol. 2011;23:434-41.

4. The Japanese HBOC Consortium. http://hboc.jp/ (2017). Accessed 23 Apr 2017

5. Nakamura S, Takahashi M, Tozaki M, Nakayama T, Nomizu T, Miki Y, et al. Prevalence and differentiation of hereditary breast and ovarian cancers in Japan. Breast Cancer. 2015;22:462-8.

6. Sugano K, Nakamura S, Ando J, Takayama S, Kamata H, Sekiguchi I, et al. Cross-sectional analysis of germline BRCA1 and BRCA2 mutations in Japanese patients suspected to have hereditary breast/ovarian cancer. Cancer Sci. 2008;99:1967-76.

7. Frank TS, Deffenbaugh AM, Reid JE, Hulick M, Ward BE, Lingenfelter B, et al. Clinical characteristics of individuals with germline mutations in BRCA1 and BRCA2: analysis of 10,000 individuals. J Clin Oncol. 2002;20:1480-90.

8. den Dunnen JT, Dalgleish R, Maglott DR, Hart RK, Greenblatt MS, McGowan-Jordan J, et al. HGVS recommendations for the description of sequence variants: 2016 update. Hum Mutat. 2016;37:564-9.

9. Evers C, Fischer C, Dikow N, Schott S. Familial breast cancer: genetic counseling over time, including patients' expectations and initiators considering the Angelina Jolie effect. PLoS ONE. 2017;12:e0177893.

10. Nagata H, Sekine M, Tsuji S, Hirai Y, Fujimoto S, Hatae M, et al. Haplotype of BRCA1 mutation alleles in Japanese ovarian and breast -ovarian cancer families: a novel method for detecting BRCA1 associated ovarian cancer. Acta Med Biol. 2002;50:33-42.

11. Sekine M, Nagata H, Tsuji S, Hirai H, Fujimoto S, Hatae M, et al. Mutational analysis of BRCA1 and BRCA2 and clinicopathologic analysis of ovarian cancer in 82 ovarian cancer families: two common founder mutations of BRCA1 in Japanese population. Clin Cancer Res. 2001;7:3144-50.

12. Kast K, Rhiem K, Wappenschmidt B, Hahnen E, Hauke J, Bluemcke B, et al. Prevalence of BRCA1/2 germline mutations in 21401 families with breast and ovarian cancer. J Med Genet. 2016;53:465-71.

13. Howlader N, Altekruse SF, Li CI, Chen VW, Clarke CA, Ries LA, et al. US incidence of breast cancer subtypes defined by joint hormone receptor and HER2 status. J Natl Cancer Inst. 2014;106: dju055.

14. Peshkin BN, Alabek ML, Isaacs C. BRCA1/2 mutations and triple negative breast cancers. Breast Dis. 2010;32:25-33.

15. Mavaddat N, Barrowdale D, Andrulis IL, Domchek SM, Eccles D, Nevanlinna $\mathrm{H}$, et al. Pathology of breast and ovarian cancers among BRCA1 and BRCA2 mutation carriers: results from the consortium of investigators of modifiers of BRCA1/2 (CIMBA). Cancer Epidemiol Biomarkers Prev. 2012;21:134-47.

16. Hori M, Matsuda T, Shibata A, Katanoda K, Sobue T, Nishimoto $\mathrm{H}$, et al. Cancer incidence and incidence rates in Japan in 2009: a study of 32 population-based cancer registries for the Monitoring of Cancer Incidence in Japan (MCIJ) project. Jpn J Clin Oncol. 2015;45:884-91.

17. Cancer Information Service, National Cancer Center. Cancer Statistics in Japan; Table download. http://ganjoho.jp/en/ professional/statistics/table_download.html (2017). Accessed 23 Apr 2017.

18. National Comprehensive Cancer Network. NCCN Clinical Practice Guideline in Oncology. Genetic/familial High-Risk Assessment: Breast and Ovarian. Version 2. https://www.nccn. org/professionals/physician_gls/pdf/genetics_screening.pdf (2017).

19. Taira N, Arai M, Ikeda M, Iwasaki M, Okamura H, Takamatsu K, et al. The Japanese Breast Cancer Society clinical practice guidelines for epidemiology and prevention of breast cancer, 2015 edition. Breast Cancer. 2016;23:343-56.

20. Brekelmans CT, Tilanus-Linthorst MM, Seynaeve C, vd Ouweland A, Menke-Pluymers MB, Bartels CC, et al. Tumour characteristics, survival and prognostic factors of hereditary breast cancer from BRCA2-, BRCA1- and non-BRCA1/2 families as compared to sporadic breast cancer cases. Eur J Cancer. 2007;43:867-76.

21. Molina-Montes E, Pérez-Nevot B, Pollán M, Sánchez-Cantalejo E, Espín J, Sánohez MJ, et al. Cumulative risk of second primary contralateral breast cancer in BRCA1/BRCA2 mutation carriers with a first breast cancer: a systematic review and meta-analysis. Breast. 2014;23:721-42.

22. Chai X, Friebel TM, Singer CF, Evans DG, Lynch HT, Isaacs C, et al. Use of risk-reducing surgeries in a prospective cohort of 1,449 BRCA1 and BRCA2 mutation carriers. Breast Cancer Res Treat. 2014;148:397-406.

23. Kim SI, Lim MC, Lee DO, Kong SY, Seo SS, Kang S, et al. Uptake of risk-reducing salpingo-oophorectomy among female BRCA mutation carriers: experience at the National Cancer Center of Korea. J Cancer Res Clin Oncol. 2016;142:333-40.

24. Zakhour M, Danovitch Y, Lester J, Rimel BJ, Walsh CS, Li AJ, et al. Occult and subsequent cancer incidence following riskreducing surgery in BRCA mutation carriers. Gynecol Oncol. 2016;143:231-5.

25. Evans DG, Baildam AD, Anderson E, Brain A, Shenton A, Vasen HF, et al. Risk reducing mastectomy: outcomes in 10 European centres. J Med Genet. 2009;46:254-8. 